\title{
Leveduras nos tratos urinário e respiratório: infecção fúngica ou não?
}

\section{Yeasts in the urinary and respiratory tracts: Is it a fungal infection or not?}

Pedrina Gonçalves Vidigal'; Terezinha Inez Estivalet Svidzinski ${ }^{2}$

unitermos Infecção fúngica invasiva

Trato urinário

Trato respiratório

\section{resumo}

O início da década de 1980 foi marcado pela emergência das infecções fúngicas hospitalares, principalmente em pacientes imunocomprometidos, resultando em graves problemas de saúde. As leveduras do gênero Candida estão entre os principais agentes desse cenário. Entretanto, as espécies não-albicans surgem não só como colonizadores, mas como patógenos responsáveis por graves infecções. Os tratos urinário (TU) e respiratório (TR) são sítios anatômicos colonizados por esses microrganismos, os quais também são susceptíveis a infecções, podendo haver disseminação hematogênica. Contudo, ainda existem controvérsias sobre a conduta mediante um achado laboratorial de leveduras nas amostras oriundas da urina ou do TR, visto que os critérios utilizados para o diagnóstico de micoses nesses sítios não estão bem estabelecidos. O objetivo deste trabalho foi abordar os aspectos referentes às dificuldades na valorização dos achados de leveduras em amostras provenientes do TU e do TR em pacientes hospitalares, além do seu envolvimento nos processos de infecções invasivas.

\section{abstract}

The beginning of 80s was noticeable by hospital fungal infections, mainly in immunocompromised patients, resulting in serious health problems. Yeasts from Candida genus are among the most important agents of these infections. However, non-albicans species appear not only as colonizers, but also as pathogens responsible for severe infections. The urinary and the respiratory tracts are anatomical sites colonized by these microorganisms and they are susceptible to infections, which may cause hematogenic dissemination. Nevertheless, there are some controversies about the medical approach towards laboratory yeast findings in samples from the urine or respiratory tract, as the criteria used for the mycoses diagnosis in these sites are still not well established. The objective of this study was to discuss the aspects related to the difficulties in evaluating yeast findings in samples from the urinary and respiratory tracts in hospitalized patients, and also their involvement in invasive infection processes. key words

Invasive fungal infection

Urinary tract

Respiratory tract 


\section{Introdução e proposição}

As espécies de Candida são consideradas patógenos oportunistas, entretanto, esses microrganismos são encontrados normalmente no corpo humano. As infecções decorrentes das espécies $C$. albicans e não-albicans apresentaram na última década aumento significativo, encontrando-se entre as principais causas de infecções nosocomiais, as quais resultam em sérios problemas de saúde pública $(19,46,90)$.

O TU é um dos sítios anatômicos mais propício para o desenvolvimento de infecções em pacientes hospitalizados, embora ainda continue sendo um problema cuja significância é duvidosa ${ }^{(35,79)}$. Por outro lado, os pulmões encontram-se envolvidos em processos infecciosos disseminados, sendo que até o momento não há uma adequada valorização dos achados de leveduras a partir de espécimes biológicos representativos do TR.

Um dilema comum enfrentado por clínicos consiste na determinação, quando na presença de um quadro de candidúria ou leveduras em amostras pulmonares, se isto de fato representa uma infecção ou se apenas resume-se a uma colonização ou, ainda, a uma contaminaçãa ${ }^{(2,36)}$.

Desta forma, a distinção entre uma autêntica infecção e uma contaminação é complicada devido à falta de critérios bem estabelecidos para diferenciar essas duas situações. Portanto, este estudo objetiva abordar o envolvimento nos processos de infecções invasivas e os aspectos relativos às dificuldades na valorização dos achados de leveduras em amostras provenientes tanto do TU como do TR em pacientes hospitalizados.

\section{Revisão de literatura}

\section{A Infecção hospitalar e o envolvimento das leveduras}

Infecção hospitalar $(\mathrm{IH})$ consiste numa das mais frequentes e importantes complicações nos pacientes submetidos à hospitalização. Segundo a instituição americana Center of Diseases Control (CDC), a IH é definida como uma infecção localizada ou sistêmica, a qual não existia no momento da admissão do paciente no hospital. Portanto, é consequência de uma reação adversa devido à presença de um agente infeccioso ou à produção de suas toxinas(32).

As IHs ocorrem no mundo todo, acometendo tanto os países desenvolvidos como os emergentes, e representam importante papel no âmbito da saúde pública. Portanto, constituem uma das principais causas de morte e de aumento da morbidade em pacientes hospitalizados, resultando assim em consideráveis gastos econômicos ${ }^{(70,72,93)}$.

A Organização Mundial da Saúde (OMS), em estudo envolvendo 47 hospitais, originários de 14 países, representando quatro continentes do mundo, revelou que $3 \%$ a $21 \%$ dos pacientes hospitalizados apresentaram IH. Adicionalmente, os maiores índices de infecções nosocomiais foram observados na Unidade de Tratamento Intensivo (UTI) ${ }^{(57)}$.

No Brasil, as estimativas prevêem que aproximadamente $5 \%$ a $15 \%$ dos pacientes durante o período de internação contraem algum tipo de infecção hospitalar ${ }^{(52)}$.

Klevens et al. ${ }^{(45)}$ conduziram um estudo nos Estados Unidos e evidenciaram que o número de infecções nosocomiais foi de aproximadamente 1,7 milhões, com 98.987 óbitos atribuídos a $\mathrm{IH}$.

Até a década de 1970, infecções sistêmicas por leveduras eram consideradas raras; as leveduras não estavam entre os agentes etiológicos mais isolados em hospitais. A partir desse momento houve importante aumento da incidência e frequência de IHs por leveduras. Espécies de Candida estão sendo consideradas como a quarta causa de infecções nosocomiais sistêmicas, representam sérios problemas, além de alta taxa de mortalidade e de questões econômicas, pelo aumento do tempo de hospitalização e, consequentemente, aumento dos custos com tratamento do paciente ${ }^{(26,47)}$.

No início da década de 1980, as infecções fúngicas emergiam como as principais causas de $\mathrm{IHs}$, acometendo principalmente pacientes imunocomprometidos ou aqueles que se encontravam hospitalizados por longos períodos devido a sérias doenças de base ${ }^{(67)}$.

As espécies de Candida compõem a microbiota de indivíduos sadios e podem encontrar-se disseminadas no meio ambiente. Usualmente, acredita-se que a maioria das pessoas apresenta uma única cepa de Candida em diferentes locais do corpo por um longo período. Entretanto, há alguns indivíduos que possuem mais de uma cepa ou espécies ao mesmo tempo, sendo este fato comumente observado em pacientes hospitalizados ${ }^{(46,62)}$.

Por outro lado, Candida spp. devem ser valorizadas, em virtude do potencial de se tornarem patógenos. Um componente crucial desta versatilidade é justamente o fato delas sobreviverem como comensais em diversos e distintos sítios anatômicos, cada uma com seus particulares estresses ambientais ${ }^{(14)}$. 
Vários fatores de virulência produzidos por esses microrganismos promovem o êxito da colonização ou da infecção invasiva dos tecidos do hospedeiro. Portanto, os fatores mais investigados são aqueles relacionados com a parede celular, a adesão, e a produção de enzimas extracelulares proteolíticas. Em primeiro lugar, porque que a parede celular propicia o crescimento do patógeno, por meio dos receptores de ligação presentes na superfície, os quais propiciam a colonização das células, bem como dos tecidos do hospedeiro. Em segundo, porque as enzimas proteolíticas estão envolvidas no processo de penetração do patógeno, enquanto as biomoléculas, neste caso as adesinas, conferem a capacidade patogênica de aderência às superfícies de mucosa, podendo resultar em colonização e infecção dos tecidos do hospedeiro ${ }^{(13,14,94)}$.

Em geral, os fungos patogênicos possuem a capacidade de superar as barreiras do sistema imunológico do hospedeiro. Vários mecanismos de defesa compartilhados são efetivos no combate aos fungos, dentre eles a produção ou liberação de oxigênio reativo e peptídeos antimicrobianos; a coestimulação de moléculas que estimulam a secreção de citocinas para desencadear a resposta imune; e o recrutamento de células efetoras para os locais de infecção através da ação de sinais inflamatórios, como a presença de citocinas, quimiocinas e componentes do sistema complemento (82). Desta forma, neutrófilos, macrófagos e monócitos são fundamentais células efetoras antifúngicas.

Embora Candida albicans possa infectar diversos sítios anatômicos do hospedeiro humano, há indícios de que a proteção imune é do tipo sítio-específico. A candidíase orofaríngea tem como fator de proteção as respostas imunes promovidas pela célula T. Por outro lado, candidíase cutânea e infecções vaginais provavelmente estejam associadas à resposta fagocítica, envolvendo os neutrófilos e os fagócitos mononucleares ${ }^{(14)}$.

Atualmente, nos Estados Unidos, as espécies do gênero Candida são, em geral, associadas à infecção nosocomial de corrente sanguínea ${ }^{(25)}$. A estimativa anual da incidência de candidemia é de seis a 10 episódios para cada 100.000 pessoas ${ }^{(60,68)}$.

No entanto, a estimativa da incidência de candidemia varia entre os países. A Finlândia detectou uma incidência de 1,9 casos para cada 100.000 pacientes hospitalizados ao ano ${ }^{(71)}$. Por sua vez, na Dinamarca, observou-se a mais elevada incidência: 11 casos para cada 100.000 pacientes hospitalares ao ano(6).

A incidência da candidemia nosocomial foi estimada em 7,6 para cada 10.000 liberações do hospital. Sendo que grande parte dos casos de candidemia hospitalar foi diagnosticada na UTI cirúrgica ${ }^{(16)}$.

Estudo realizado no norte de Taiwan detectou que Candida spp. são os principais patógenos de IH da corrente sanguínea ${ }^{(37)}$.

De acordo com Surveillance and Control of Pathogens of Epidemiological Importance (SCOPE), num estudo realizado durante o período de 1995 a 2002, constatou-se que Candida spp. foi a quarta causa mais comum de infecções nosocomiais de corrente sanguínea, correspondendo a $9 \%$ dessas infecções. A incidência de candidemia é mais elevada em UTIs; nesta unidade, candidemia é associada a 10,1\% das infecções sanguíneas, enquanto a relação na enfermaria foi de $7,9 \%{ }^{(97)}$.

Em 2005, na Itália, um estudo também corroborou que a frequência da candidemia é maior em UTIs, quando comparada aos centros cirúrgicos ou às enfermarias ${ }^{(51)}$. Os índices apresentados foram de 15,8/10.000 pacientes/dias em UTIs, enquanto para a enfermaria e para os centros cirúrgicos foram estimados em 0,15/10.000 e 0,69/10.000 pacientes/ dias, respectivamente.

Diante disso, fica evidente que a candidemia hospitalar implica em elevados índices de morbidade e mortalidade. Gudlaugsoon et al. ${ }^{(34)}$, reavaliando a mortalidade decorrente da candidemia hospitalar 15 anos após um estudo retrospectivo, demonstraram que a mortalidade bruta foi de $61 \%$, considerando uma mortalidade atribuída equivalente a $49 \%$, quando comparada com o grupo controle.

Falagas et al. ${ }^{(28)}$ também concluíram a existência de um considerável grau de associação entre candidemia e mortalidade atribuída. Segundo os autores, o óbito não pode ser relacionado somente com a presença de outras comorbidades.

Entretanto, Pfaller e Diekema ${ }^{(68)}$, avaliando a taxa de mortalidade durante o período de 1991 a 2003, presenciaram um discreto decréscimo associado à candidemia ao longo deste tempo.

Uma vez que a candidemia está relacionada com longo período de permanência em UTIs, implica em aumento considerável no custo de manutenção deste paciente. Estima-se que nos Estados Unidos os gastos com tratamentos sejam de dois a quatro bilhões de dólares anuais ${ }^{(95)}$. De acordo com Olaechea et al.(63), tanto a colonização quanto a infecção causadas por Candida spp. demandam grande impacto econômico, devido ao longo período em UTI e no próprio hospital. Segundo os mesmos autores, os pacientes que foram liberados do hospital devido à colonização e à infecção 
por Candida spp. resultaram num impacto econômico de US $\$ 12.908,00$ e US $\$ 21.075$, respectivamente.

Os aspectos clínicos da candidemia não são específicos. Segundo Aquino et al. ${ }^{(5)}$, o principal sintoma clínico observado foi febre (87\%), enquanto foram detectadas especificamente lesões cutâneas e oculares em 13,1\% e $3,9 \%$ dos pacientes, respectivamente. Já em outro estudo realizado, os sintomas mais frequentes foram febre com rigidez, taquicardia e hipotensão(11).

No entanto, na prática clínica, há alguns relatos sobre o envolvimento de diferentes órgãos em casos de candidemia, tais como o sistema nervoso central (SNC), o qual apresenta elevada frequência em neonatos prematuros; o coração, desencadeando na infecção do tecido endocárdico; as articulações, principalmente as maiores, comumente observadas em crianças; e raramente o fígado e o baço(22).

A candidemia prolongada é grave, precisa ser tratada adequadamente e é confirmada por hemoculturas positivas que sugerem a presença de foco ou fonte persistente, como cateter venoso central (CVC), abscessos, tromboflebite e endocardite ${ }^{(96)}$.

Em geral, os fatores de risco para os episódios de candidemia são neutropenia severa, cirurgia prévia; insuficiência renal aguda (IRA); nutrição parenteral; e uso de instrumentação invasiva, como o $\operatorname{CVC}^{(8,18,55)}$. Entretanto, a análise multivariada evidenciou em estudo que o tempo de hospitalização, a presença de CVC, bem como os episódios de bacteremia, o tratamento de nutrição parenteral e a insuficiência renal aguda são variáveis independentes associadas ao desenvolvimento de sepse por espécies de Candida $^{(8)}$.

Por outro lado, Chow et al.(18) mostraram que além desses riscos, o número de dias de hemodiálise, assim como cirurgias prévias, também constitue importante variável associada à candidemia causada não só por Candida albicans, mas também pelas outras espécies deste gênero. Segundo esses autores, o número de dias com ventilação mecânica e o número de dias com CVC foram altamente correlacionados.

Tumbarello et al. ${ }^{\left({ }^{89)}\right.}$ detectaram que os fatores de risco mais comuns estavam correlacionados com comorbidades, como o câncer em órgão sólido (40,1\%), seguida do diabete mellitus (DM) $(19,7 \%)$ e das doenças hepáticas (10,2\%). Os autores destacaram, ainda, o emprego da terapia antifúngica inadequada e a habilidade de formação de biofilme pelas espécies de Candida, como fortemente associados à elevada mortalidade nestes pacientes.
Outros fatores atuam na indução de imunossupressão, assim como a utilização de corticosteroides, de imunomodeladores; a quimioterapia; a desnutrição; a condição de malignidade e o quadro de neutropenia; queimaduras também favorecem o desenvolvimento de infecção fúngica ${ }^{(30)}$.

Além disso, instrumentos médicos como as próteses, os implantes, as sondas, os tubos endotraqueais, os marca-passos cardíacos e os diversos tipos de cateteres têm revelado capacidade de auxiliar no processo de colonização e na formação de biofilmes ${ }^{(73)}$. Biofilmes formados nestes instrumentos exercem a função de reservatório ou de fonte de leveduras para futuras infecções contínuas. A organização estruturada e a fixação destes microrganismos em matriz de material extracelular podem gerar um impacto negativo sobre o paciente, acarretando insuficiência dos mesmos $^{(24)}$.

A infecção fúngica invasiva consiste no aumento da biomassa do patógeno, o qual coloniza determinado sítio anatômico. Portanto, a infecção se agrava à medida que este patógeno se dissemina do local inicial da colonização, promovendo a sua penetração em barreiras defensivas do hospedeiro. Consequentemente, ocorrem danos tissulares e, casualmente, desencadeia-se um processo sistêmico( ${ }^{(54)}$.

Entretanto, para ser considerada infecção, o caso requer pelo menos uma dessas características: documentação de hemocultura positiva com confirmação do crescimento de Candida spp.; verificação oftalmológica com endoftalmite em paciente que apresente quadro de sepse; e isolamento de Candida spp. de amostras biológicas significativas (líquido pleural, líquido ascítico, líquido pericárdico) ou provenientes da cavidade abdominal após ato cirúrgico(63).

Segundo alguns pesquisadores, grande parte das candidemias são originalmente endógenas, sendo portanto adquiridas pela colonização prévia de locais anatômicos que atuam como reservatórios, tais como a cavidade oral, o trato gastrointestinal $(\mathrm{TGl})$, a vagina ou a pele. No entanto, há também indícios de que as infecções exógenas e as infecções cruzadas decorrentes da má-higienização das mãos de profissionais da área médica possam ocorrer ${ }^{(1,44,87)}$. A transmissão de um paciente infectado para outro pode ocorrer em curto espaço de tempo, entretanto, as cepas de Candida albicans podem sobreviver e resistir por um longo período fora do organismo ${ }^{(76)}$.

\section{Vias de acesso à candidemia - candidúria e candidíase pulmonar}

O TU consiste no sítio anatômico mais propício para o desenvolvimento de infecções em pacientes hospitalizados, 
embora ainda permaneça como um problema de duvidosa significância ${ }^{35,79}$. Ainda que a maioria dessas infecções seja de origem bacteriana, estima-se que pelo menos $10 \%$ delas tenham como principal agente etiológico os fungos, sendo as espécies do gênero Candida as mais isoladas ${ }^{(75,84)}$. Há dados que evidenciam o isolamento de Candida em 22\% das amostras de urina proveniente de pacientes admitidos em UTIs(3).

As últimas duas décadas foram marcadas pelo aumento das infecções fúngicas nosocomiais do TU, possivelmente devido aos vários fatores predisponentes, os quais são relacionados com ocorrência de candidúria ${ }^{(85)}$.

O termo candidúria pode ser expresso como o crescimento de leveduras do gênero Candida em culturas de urina, coletadas por meio de técnicas adequadas ${ }^{(19)}$.

A recuperação de Candida spp. em amostras de urina expõe os clínicos a um grande desafio em virtude da amplitude de possibilidades clínicas. Dentre elas, destacam-se a pielonefrite; a cistite; a ocorrência de uma propagação hematogênica a partir do córtex renal, favorecendo o curso da candidíase disseminada assintomática ou sintomática; e, por fim, fato este mais provável, a colonização de sítios anatômicos como a bexiga e o períneo, ou ainda a presença de um cateter urinário. Portanto, nem a presença de sintomas e sinais de infecção ou ainda a contagem de colônias na urina fornecem subsídios suficientes para a interpretação da importância clínica da candidúria ${ }^{(41,50)}$.

O curso natural da candidúria ainda não está claramente estabelecido. Por outro lado, a colonização do TU deve ser observada e acompanhada com mais atenção, uma vez que é considerada um evento comum em pacientes hospitalizados $^{(53)}$.

Os fatores de riscos associados à candidúria usualmente referem-se à administração de antibióticos; terapia imunossupressiva; a procedimentos cirúrgicos prévios; à idade avançada; ao sexo feminino; longo período de hospitalização; a comorbidades, em especial o DM; e à utilização de instrumentos urinários invasivos, tais como cateteres ${ }^{(12,50)}$. A análise de regressão logística multivariada comprovou que o uso de antibióticos e o nível de glicose superior a $180 \mathrm{mg} / \mathrm{dl}$ isoladamente já são parâmetros associados à candidúria ${ }^{(66)}$.

A primeira linha de defesa do corpo humano consiste nas barreiras mecânicas, como a pele e o epitélio das mucosas. A barreira física do TR superior é o epitélio, o qual é responsável pela incorporação dos potenciais patógenos em um muco e a sua consequente eliminação. Entretanto, uma segunda linha de defesa é exigida quando essa mucosa perde a sua integridade, promovendo não só a realização de ações não-específicas, como também a ativação do sistema imune. Portanto, uma modificação em quaisquer mecanismos de defesa permite que patógenos oportunistas possam infectar o corpo ${ }^{(29)}$.

Castellani apud Morin et al. ${ }^{(15)}$ foi o primeiro a descrever uma afecção broncopulmonar em trabalhadores de chá provenientes da região de Ceylon. A partir da administração do pó de chá no TR superior em roedores "cobaias" (Cavia porcellus) durante vários meses, o autor os induziu às lesões pulmonares extensivas semelhantes àquelas isoladas nos trabalhadores.

A colonização do TR, desencadeada pelas espécies do gênero Candida, é comum em pacientes que utilizam a ventilação-mecânica (VM) por um período superior a dois dias. Isto ocorre em virtude da disseminação hematogênica pulmonar ou pela aspiração de conteúdos colonizados de origem orofaríngea ou gástrica ${ }^{(59)}$. É importante, também, o período prolongado de permanência na UTI, bem como de internação(7).

Além disso, Delisle et al.(21) demonstraram, a partir da análise de regressão, que há uma associação significativa entre a colonização do trato respiratório por Candida com a mortalidade hospitalar.

Frequentemente, Candida spp. são encontradas no escarro e nos aspirados dos tubos de secreção endotraqueal, embora o envolvimento desses agentes infecciosos na causa de doenças pulmonares ainda permaneça um dilema clínico. A infecção por Candida é confirmada histopatologicamente, e a invasão pulmonar usualmente não apresenta significância clínica comum. Em alguns casos de envolvimento pulmonar extensivo, a pneumonite ocorre comumente em pacientes terminais ${ }^{(23)}$.

Rotter e Staib ${ }^{(78)}$ realizaram um estudo sobre pneumonia causada por Candida tropicalis, abordando os aspectos clínicos bem como o tratamento. Já na década de 1960, os estudos sobre candidíase pulmonar limitaram-se a poucos, como os conduzidos na Itália, Alemanha e Espanha, respectivamente ${ }^{(9,43,48,80)}$.

Masur et al.(56), num período de dois anos, avaliaram 30 pacientes provenientes do Memorial Hospital e New York Hospital, os quais apresentavam evidências histológicas de infecção pulmonar desencadeada por Candida. Os autores concluíram que aproximadamente $33 \%$ das infecções atingiram os pulmões por meio da disseminação hematológica, enquanto $54 \%$ foram pela aspiração. Além 
disso, observou-se que a maioria dos pacientes apresentava doença neoplásica maligna.

Rose e Sheth ${ }^{(77)}$, verificando a correlação clínica e patológica da candidíase pulmonar, conduziram um estudo prospectivo num período de 13 anos. A candidíase pulmonar foi diagnosticada em 11 pacientes, dos quais nove apresentaram disseminação hematológica, enquanto dois revelaram pneumonia por aspiração. Adicionalmente, ressaltam que a candidíase pulmonar normalmente surge a partir da implantação de um foco infeccioso durante a disseminação hematológica. Os mesmos autores detectaram, ainda, que a utilização do cateter venoso constituiu a fonte para o desenvolvimento da candidemia em seis pacientes.

Estima-se que em pacientes que apresentam neoplasia, mais de $20 \%$ das infecções fúngicas nosocomiais sejam de origem pulmonar ${ }^{(39)}$.

Shen et al. ${ }^{(83)}$ avaliaram os fatores etiológicos, o diagnóstico e a terapia da infecção fúngica pulmonar relacionados com as doenças hematológicas. Os autores observaram, então, a presença de Candida albicans em 71,42\% dos casos estudados.

Xie et al. ${ }^{(98)}$ avaliaram o impacto da infecção fúngica invasiva (IFI) em pacientes cirúrgicos doentes com sepse severa. Os autores observaram que $28,3 \%$ dos pacientes avaliados apresentaram IFI, sendo a espécie $C$. albicans a mais isolada (58\%), seguida por C. tropicalis (17\%) e C. glabrata (15\%). Em adição, também revelaram que o órgão mais acometido pela IFI foi o pulmão.

\section{Cenário microbiológico das espécies do gênero Candida}

Embora a Candida albicans ainda permaneça como umas das espécies mais isoladas ${ }^{(65)}$, ultimamente nota-se a emergência de outras, como C. tropicalis, C. parapsilosis, C. glabrata, C. krusei, C. lusitaniae e C. guillermondii(31). Tumbarello et al. ${ }^{\left({ }^{89)}\right.}$ encontraram como isolados mais frequentes de candidemias as espécies $C$. tropicalis $(71,4 \%)$, C. glabrata $(23,1 \%)$, C. albicans $(22,6 \%)$ e C. parapsilosis $(21,8 \%)$. Acompanhando as alterações quanto às espécies, observam-se variações na susceptibilidade aos antifúngicos (anfotericina B e derivados azólicos). Consequentemente, este fato eleva as complicações para a escolha do tratamento a ser adotado inicialmente ${ }^{(40,64,74,88,91,97)}$. As razões para essa inversão, com relação à distribuição das espécies, ainda não foram completamente esclarecidas.

Vários trabalhos conduzidos em diferentes hospitais brasileiros também detectaram mudança na distribuição dos agentes etiológicos nas IHs. Colombo et al. ${ }^{(20)}$ revelaram que dos 145 episódios de candidemias avaliados, apenas $37 \%$ foram desencadeados por C. albicans, enquanto os $63 \%$ restantes tiveram como agente causal espécies nãoalbicans. Em outro relato, as fungemias presenciadas em pacientes oncológicos tiveram como espécie mais frequente a C. tropicalis, enquanto C. albicans representou apenas $15 \%$ desses $\operatorname{casos}^{(61)}$.

\section{Diagnóstico laboratorial para detecção de leveduras}

Um problema frequente enfrentado por clínicos consiste em determinar quando a presença de candidúria representa, de fato, infecção do TU ou se simplesmente é uma colonização ou contaminação(2). Desta forma, a distinção entre infecção autêntica e contaminação é complicada e ainda carece de critérios padronizados.

A contaminação da urina é um evento normal e ocorre especialmente em duas situações: quando a coleta não é adequada, frequente em pacientes sob cateterização urinária, ou em mulheres, pela elevada colonização da região vulvovaginal(50).

Atualmente, ainda não há metodologia fidedigna e segura para diferenciar colonização de infecção na presença de uma candidúria detectada em laboratório ${ }^{(10)}$. Para alguns, somente a simples presença de leveduras em uroculturas ou o exame direto já é suficiente para definir infecção(81). No entanto, em alguns estudos a quantificação torna-se um dos critérios para a obtenção de tal definição, havendo ainda controvérsias para se estabelecer um valor-padrão. Alguns grupos de pesquisa acreditam que a contagem referente a $1.000 \mathrm{UFC} / \mathrm{ml}^{(42,49,86,92)}$ é satisfatória para o diagnóstico de candidúria, enquanto para outros este valor pode ser igual ou superior a $10.000 \mathrm{UFC} / \mathrm{ml}^{(3,33,38)}$.

No entanto, a infecção urinária pode ser caracterizada pela elevada colonização, a partir de uma contagem superior a $10.000 \mathrm{UFC} / \mathrm{mL}$, demonstrando, no máximo, a presença de duas espécies distintas. Além disso, alguns sintomas, como febre, urgência miccional, polaquiúria, disúria ou tensão na zona suprapúbica também compõem o quadro clínico de infecção urinária por Candida spp. ${ }^{(32)}$.

$\mathrm{O}$ isolamento de Candida a partir de culturas de escarro, aspirados endotraqueais, amostras broncoscópicas, aspirados de punção percutânea do pulmão, ou até mesmo de tecido pulmonar pode representar somente colonização da árvore traqueobrônquica. Em adição, o valor de culturas quantitativas com amostras originárias 
do trato respiratório para o diagnóstico de pneumonia, em decorrência das espécies de Candida, ainda permanece desconhecido ${ }^{(27)}$.

Normalmente, as culturas resultantes de materiais, como o sangue, o líquido encefaloraquidiano (LCR), o líquido sinovial, o abscesso coletado esterilmente ou qualquer outro espécime cirúrgico estéril, são consideradas representativas para o diagnóstico de candidíase ${ }^{(65)}$. A hemocultura ainda prevalece como padrão-ouro para o diagnóstico de candidemia. Entretanto, a sensibilidade deste exame é de somente $50 \%$ a $60 \%$, o que pode propiciar um diagnóstico falso-negativo e, consequentemente, resultar numa subestimação do impacto da mesma ${ }^{(46,99)}$. Sendo assim, em várias ocasiões o médico clínico é forçado a conduzir um tratamento empírico com base nos fatores de riscos e na suspeita clínica. Em virtude dessas e de outras razões, ainda hoje nem sempre é realizada a investigação laboratorial da candidemia, o que é lamentável, pois, em geral, as hemoculturas de amostras com C. albicans, C. parapsilosis e C. tropicalis tornam-se positivas num período de um a dois dias ${ }^{(4,60)}$. Assim sendo, considerando que a escolha inicial do tratamento antifúngico é de extrema importância, dados relacionados com a espécie e a susceptibilidade aos antifúngicos contribuiriam muito para a melhora da eficácia da terapia ${ }^{(65)}$.

De fato, os recursos laboratoriais atualmente disponíveis são limitados, visto que a sensibilidade das hemoculturas é baixa, embora seja inquestionável a sua capacidade de definir infecção. Além disso, atualmente não há protocolos padronizados que sejam reconhecidos e que possam oferecer subsídios capazes de predizer precocemente o risco da presença de leveduras detectadas em amostras provenientes do TU ou do TR, mesmo considerado a probabilidade de uma colonização evoluir em infecções de alta morbidade e mortalidade em determinados casos clínicos. Estudos prévios já haviam demonstrado a importância da colonização por Candida. Isto é elucidado pelo fato de a patogênese da infecção causada por este agente infeccioso evoluir progressivamente a partir da colonização local, acarretando em ampla disseminação e, consequentemente, invasão ${ }^{(69,74)}$. Recentemente, Cheng et al. ${ }^{(16)}$ observaram que $79,5 \%$ dos casos de candidemia foram precedidos pela colonização, considerando que as taxas mais elevadas de colonização foram detectadas na urina (40\%) e no CVC (35\%).

Portanto, a caracterização da diversidade genética das leveduras C. albicans e não-albicans torna-se importante em virtude do conhecimento da estrutura genética, que, consequentemente, irá auxiliar a equipe médica a adotar a terapia mais adequada para cada paciente. Assim, a epidemiologia molecular pode também determinar se as infecções detectadas são recorrentes ou se houve recontaminação por outra cepa. Adicionalmente, os métodos de genotipagem são considerados importantes na identificação da fonte de contaminação, tais como os instrumentos invasivos, como o cateter; as mãos dos profissionais que trabalham em hospital; ou ainda os sítios endógenos do corpo ${ }^{(17)}$.

\section{Considerações finais}

Embora alguns profissionais da saúde demonstrem preocupação com as doenças fúngicas no ambiente hospitalar, esta realidade tanto em países emergentes como em países desenvolvidos ainda não está bem estabelecida ${ }^{(70,93)}$.

Um diagnóstico correto propiciará o tratamento adequado para o paciente, além de representar melhora no quadro das taxas de mortalidade decorrentes destas infecções. Portanto, a padronização de conduta tomada por clínicos e laboratoristas torna-se fundamental para a garantia do diagnóstico adequado, bem como da qualidade de vida de pacientes críticos, sendo indispensáveis os estudos que visem contribuir para a valorização dos quadros de colonização por leveduras. Desta forma, os mesmos poderão, em curto ou médio prazos, contribuir para a implantação de protocolos universais que proporcionem a rápida e eficiente tomada de decisões quanto ao manejo dos pacientes criticamente doentes.

\section{Referências}

1. AHMAD, S. et al. Epidemiology of Candida colonization in an intensive care unit of teaching hospital in Kuwait. Med Mycol, v. 41, p.487-493, 2003.

2. AKALM, H. et al. Persistence of candiduria in ICU catheterized patients is not linked to adherence and proteolytic activities of Candida strains. Intes Care Med, v. 30 , p. $972-975,2004$
3. ALVAREZ-LERMA, F. et al. Candiduria in critically ill patients admitted to intensive care medical units. Care Med, v. 29, p. 1069-1076, 2003.

4. ANAISSIE, E.; McGINNIS, M.R.; PFALLER, M.A. Clinical Mycology. 1st ed. Philadelphia: Elsevier Science, 2003. p.195-239.

5. AQUINO, V.R. et al. Prevalence, susceptibiliy profile for fluconazole and risk foactors for candidemia in a tertiary 
care hospital in Southen Brazil. Braz J Infects Dis, v.9, p. 411-418, 2005.

6. ARENDRUP, M.C. et al. Seminational surveillance of fungemia in Denmark: notably high rates of fungemia and numbers of isolates with reduced azole susceptibility. J Clin Microbiol, v.43, p. 4434-4440, 2005.

7. AZOULAY, E. et al. Candida colonization of the respiratory tract and subsequent Pseudomonas ventilatorassociated pneumonia. Chest, v. 129, p. 110-117, 2006.

8. BASSETTI, M. et al. Incidence, risk factors, and predictors of outcome of candidemia. Survey in 2 Italian university hospitals. Diagn Microbiol Infect Dis, v.58, p. 325331, 2007.

9. BIDER, F.; FIORA, L. A case of pulmonary candidosis treated with amphotericin B. Minerva Med, v. 56, p. 3364-3367, 1965.

10. BLOT, S. et al. Is Candida really a threat in the ICU? Curr Opin Crit Care, v. 14, p. 601-604, 2008.

11. BREGENZER, T. et al. Clinical aspects of candidemia, a 6-year retrospective study. Schweiz Med Wochenschr, v. 126, p. 1829-1833, 1996.

12. BUKHARY, Z.A. Candiduria: a review of clinical significance and management. Saudi J Kidney Dis Transplant, v. 19, p. 350-360, 2008.

13. CALDERONE, R.A.; BRAWN, P.C. Adherence and receptor relationships of Candida albicans. Microbiol Rev, v. 55, p. 1-20, 1991.

14. CALDERONE, R.A.; FONZI, W.A. Virulence factors of Candida albicans. Trends Microbiol, v. 9, p. 327335, 2001.

15. CASTELLANI, A. Lancet, v.1, p. 13, 1912. Quoted by Langeron apud MORIN, J.E.; LEBLOND, S.; FISET, P. Bronchopulmonary candidosis. Can Med Assoc J, v. 65, p. 115-118, 1951.

16. CHENG, Y-R et al. Risk factors for candidemia-related mortality at a medical Center in Taiwan. J Microbiol Immunol Infect, v. 39, p. 155-161, 2006.

17. CHONG, P.P. et al. Recurrent candidaemia in a neonate with Hirschsprung's disease: fluconazole resistance and genetic relatedness of eight Candida tropicalis isolates. J Med Microbiol, v. 55, p. 423-428, 2006.

18. CHOW, J.K. et al. Risk factors for albicans and non-albicans candidemia in the intensive care unit. Crit Care Med, v.36, p. 1993-1998, 2008.

19. COLOMBO, A.L.; GUIMARÃES, T. Candidúria: uma abordagem clínica e terapêutica. Rev Soc Bras Med Trop, v. 40, p. 332-337, 2007.

20. COLOMBO, A.L. et al. High rate of non-albicans candidemia in Brazilian tertiary care hospitals. Diagn Microbiol Infect Dis, v.34, p. 281-286, 1999.

21. DELISLE M-S et al. The clinical significance of Candida colonization of respiratory tract secretions in critically ill patients. J Crit Care, v. 23, p. 11-17, 2008.

22. DIGNANI, M.C.; SOLOMKIN, J.S.; ANAISSIE, E. Candida. In: ANAISSIE, E.; McGINNIS, M.R.; PFALLER, M.A. (eds). Medical Mycology. $1^{\text {a }}$ ed. Churchill Livingstone, Filadélfia, p. 195-239, 2003.

23. DISMUKES, W.E.; PAPPAS, P.G.; SOBEL, J.D. Clinical Mycology. United States, Oxford University Press, 2003. p.159.
24. DOUGLAS, L.J. Medical importance of biofilms in Candida infections. Rev Iberoam Micol, v. 19, p. 139-143, 2002.

25. EDMOND, M.B. et al. Nosocomial bloodstream infections in the United States hospitals: a three-year analysis. Clin Infect Dis, v. 29, p. 239-244, 1999.

26. EGGIMANN, P.; GARBINO, J.; PITTET, D. Epidemiology of Candida species infections in critically ill nonimmunosuppressed patients. Lancet Infect Dis, v. 3, p. 685-702, 2003.

27. EL-EBIARY, M. et al. Significance of the isolation of Candida species from respiratory samples in critically ill, non-neutropenic patients. An Immediate postmortem histologic study. Am J Respir Crit Care Med, v. 156, p. 583-590, 1997.

28. FALAGAS, M.E.; APOSTOLOU, K.E.; PAPPAS, V.D. Attributable mortality of candidemia: a systematic review of matched cohort and case-control studies. Eur J Microbiol Infect Dis, v. 25, p. 419-425, 2006.

29. FORREST, L.A.; WEED, H. Candidalaryngitis appearing as leukoplakia and gerd. J Voice, v. 12, p. 91-95, 1998.

30. FRIDKIN, S.K.; JARVIS, W.R. Epidemiology of nosocomial fungal infections. Clin Microbiol Rev, v. 9, p. 499511, 1996.

31. GALVAN, B.; MARISCAL, F. Epidemiologia de la candidemia en UCl. Rev. Iberoam Micol, v. 23, p. 12-15, 2006.

32. GARNER, J.S. et al. CDC definitions for nosocomial infections. In: Olmsted RN, ed.: APIC Infection Control and Applied Epidemiology: Principles and Practice. St. Louis: Mosby, 1996, pp.A1-A-20.

33. GUBBIS, P.O.; PISCITEKKI, S.C.; DANZIGER, L.H. Candidal urinary tract infections: a comprehensive review of their diagnosis and management. Pharmacotherapy, v. 13, p. 110-117, 1993.

34. GUDLAUGSOON, O. et al. Attributable mortality of nosocomial candidemia, revisited. Clin Infect Dis, v. 37, p. 1172-1177, 2003.

35. GULER, S. et al. Risk factors for nosocomial candiduria. Saudi Med J, v. 27, p. 1706-1710, 2006.

36. HARON, E. et al. Primary Candida pneumonia. Medicine, v. 72, p. 137-142, 1993.

37. HSUEH, P.R. et al. Antimicrobial drug resistance in pathogens causing nosocomial infections at a university hospital in Taiwan, 1981-1999. Emerg Infect Dis, v. 8, p. 63-68, 2002.

38. JACOBS, L.G. et al. Oral fluconazole compared with bladder irrigation with amphotericin $B$ for treatment of fungal urinary tract infections in elderly patients. Clin Infect Dis, v. 22, p. 30-35, 1996.

39. JIANG, Y. et al. Clinical analysis of nosocomial pulmonary fungal infection in patients with cancer. Ai Zheng, v. 23, p. 1707-1709, 2004.

40. KAO, A.S. et al. The epidemiology of candidemia in two United States cities: results of a population-based active surveillance. Clin Infect Dis, v. 29, p. 11641170, 1999.

41. KAUFFMAN, C.A. Candiduria. Clin Infect Dis, v. 41, S371S376, 2005.

42. KAUFFMAN, C.A. et al. Prospective multicenter surveillance study of funguria in hospitalized patients. Clin Infect Dis, v. 30, p. 14-18, 2000. 
43. KAUR, H.; CHUGH, T.D.; CHITKARA, N.L. Bronchopulmonary candidosis. Indian J Chest Dis, v. 11, p. 207-212, 1969.

44. KHAN, Z.U.; CHANDY, R.; METWALI, K.E. Candida albicans strain carriage in patients and nursing staff of an intensive care unit: a study of morphotypes and resistotypes. Mycoses, v. 46, p. 479-486, 2003.

45. KLEVENS, R.M. et al. Estimating health care-associated infections and deaths in U.S. hospitals. Public Health Rep, v. 122, p. 160- 166, 2002.

46. KLOTZ, S.A. et al. Polymicrobial bloodstream infections involving Candida species: analysis of patients and review of the literature. Diagn Microbiol Infect Dis, v. 59, p. 401-406, 2007.

47. KRCMERY, V.; BARNES, A.J. Non-albicans Candida spp. causing fungaemia: pathogenicity and antifungal resistance. J Hosp Infect, v. 50, p. 243-260, 2002.

48. LAMPE, W. On the therapy of generalized candidiasis of both lungs. Med Welt, v. 5, p. 282-286, 1967.

49. LEU, H.S.; HUANG, C.T. Clearance of funguria with short-course antifungal regimens: a prospective, randomized, controlled study. Clin Infect Dis, v. 20, p. 1152-1157, 1995.

50. LUNDSTROM, T.; SOBEL, J. Nosocomial Candiduria: a review. Clin Infect Dis, v. 32, p. 1602-1607, 2001.

51. LUZZATI, R. et al. Secular trends in nosocomial candidaemia in non-neutropenic patients in an Italian tertiary hospital. Clin Microbiol Infect, v. 1, p. 908913, 2005.

52. MACHADO, A. et al. Prevenção da Infecção Hospitalar. In: Projeto Diretrizes: Associação Médica Brasileira e Conselho Federal de Medicina. Agos. 2001.

53. MAGILL, S. S. et al. The association between anatomic site of Candida colonization, invasive candidiasis, and mortality in critically ill surgical patients. Diagn Microbiol Infect Dis, v. 55, p. 293-301, 2006.

54. MAGRYS, A. et al. The prognostic and diagnostic markers of invasive candidiasis in patients during chemotherapy. Pol J of Microbiol, v. 54, p. 207-213, 2005.

55. MASCHMEYER, G. The changing epidemiology of invasive fungal infections: new threats. Int J Antimicrob Agents, suppl. 27, p. 3-6, 2006.

56. MASUR, H.; ROSEN, P.P.; ARMSTRONG, D. Pulmonary disease caused by Candida species. Am J Med, v. 63, p. 914-925, 1977.

57. MAYON-WHITE, R.T. et al. An international survey prevalence of hospital-acquired infection. J Hosp Infect, v. 11, suppl. A, p. 43-48, 1988.

58. MORGAN, J. Global trends in candidemia: review of reports from 1995-2005. Curr Infect Dis Rep, v. 7, p. 429-439, 2005.

59. MURRAY, P.R.; VANSCOY, R.E.; ROBERTS, G.D. Should yeasts in respiratory secretions be identified? Mayo Clin Proc, v. 52, p. 42-45, 1977.

60. NODA, T. et al. Basic and clinical evaluation of lysis centrifugation in candidemia. Kasenshhogaku Zassi, v. 69 , p. $145-150,1995$.

61. NUCCI, M. et al. Fungemia in cancer patients in Brazil: predominance of non-albicans species. Mycopathologia, v. 141, p. 65-68, 1998.
62. ODDS, F.C. Candida infections: an overview. Crit Rev Microbiol, v. 32, p. 123-129, 1987.

63. OLAECHEA, P.M. et al. Economic impact of Candida colonization and Candida infection in the critically ill patient. Eur J Microbiol Infect Dis, v. 23, p. 323-330, 2004.

64. PAPPAS, P.G. et al. A prospective observation study of candidemia: epidemiology, therapy, and influences on mortality in hospitalized adult and pediatric patients. Clin Infect Dis, v. 37, p. 634-643, 2003.

65. PATEL, M. et al. Initial management of candidemia at an academic medical center: evaluation of the IDSA guidelines. Diagn Microbiol Infect Dis, v. 52, p. 29-34, 2005.

66. PAUL, N. et al. Factors associated with candiduria and related mortality. J Infect, v. 55, p. 450-455, 2007.

67. PAULA, C.R. et al. Infecção hospitalar fúngica: experiência em hospitais públicos de São Paulo. Prática hospitalar, v. 52, p. 63-66, 2007.

68. PFALLER, M.A.; DIEKEMA, D.J. Epidemiology of invasive candidiasis: a persistent public health problem. Clin Microbiol Rev, v. 20, p. 133-163, 2007.

69. PITTET, D. et al. Candida colonization and subsequent infections in critically ill patients. Ann Surg, v. 220, p. 751-758, 1994.

70. PLOWMAN, R. et al. The socio-economic burden of hospital-acquired infection. London, Public Health Laboratory Service and the London School of Hygiene and Tropical Medicine, 1999.

71. POIKONEN, E. et al. Candidemia in Finland, 1995-1999. Emerg Infect Dis, v. 9, p. 985-990, 2003.

72. PONCE-DE-LEON, S. The needs of developing countries and the resources required. J Hosp Infect, v. 18, S376S381, 1991.

73. RAMAGE, G. et al. Candida biofilms: an update. Eukaryotic Cell, v. 4, p. 633-638, 2005.

74. RANGEL-FRAUSTO, M.S. et al. National epidemiology of mycoses survey (NEMIS): variations in rates of bloodstream infections due to Candida species in seven surgical intensive care units and six neonatal intensive care unit. Clin Infec Dis, v. 28, p. 253-258, 1999.

75. RIVETT, A.G.; PERRY, J.A.; COHEN, J. Urinary candidiasis: a prospective study in hospitalized patients. Urol Res, v. 14, p. 183-186, 1986.

76. RODERO, L. et al. Trasmisión nosocomial de Candida albicans en recién nacidos. Ver Argent Microbiol, v. 32, p. 179-184, 2000.

77. ROSE, H.D.; SHETH, N.K. Pulmonary candidiasis. A clinical and pathological correlation. Arch Intern Med,v. 138, p. 964-965, 1978.

78. ROTTER, W.; STAIB, F. Candida tropicalis pneumonia; treatment and course. Dtsch Med Wochenschr, v. 83, p. 2285-2288, 1958.

79. SCHABERG, D.R. et al. Major trends in the microbial etiology of nosocomial infection. Am J Med, v. 91, suppl. 3B, p. 72-75, 1991.

80. SCHOCKY, S. Pulmonary candidiasis. Med Klin, v. 61, p. 492-497, 1966.

81. SELLAMI, A. et al. La candidurie en miliey de réanimation: signification et intérêt de la numération des levares dans les urines. Ann Fr Anesth Reanim, v. 25, p. 584-588, 2006. 
82. SHOHAM, S.; LEVITZ, S.M. The immune response to fungal infections. Br J Haematol, v. 129, p. 569-582, 2005.

83. SHEN, Q. et al. Pulmonary fungal infection in malignant hematological diseases: an analysis of 14 cases. Zhongguo Shi Yan Xue Ye Xue Za Zhi, v. 13, p. 11251127, 2005.

84. SOBEL, J.D. et al. Candiduria: a randomized double-blind study of treatment with fluconazole and placebo. Clin Infect Dis, v. 30, p. 19-24, 2000.

85. SOBEL, J.D. Controversies in the diagnosis of candiduria: What is the critical colony count? Infect Dis, v. 4, p. 81-83, 2002.

86. SOBEL, J.D. Practice guidelines for the treatment of fungal infections. For mycoses Study Group. infectious diseases society of America. Clin Infect Dis, v. 30, p. 652, 2000.

87. TIRABOSCHI, I.N. et al. Brote de candidemia por Candida albicans en neonatología. Rev Iberoam Micol, v. 24, p. 263-267, 2007.

88. TRICK, W.E. et al. Secular trend of hospital-acquired candidemia among intensive care unit patients in the United States during 1989-1999. Clin Infect Dis, v. 35, p. 627-630, 2002.

89. TUMBARELLO, M. et al. Biofilm production by Candida species and inadequate antifungal therapy as predictors of mortality for patients with candidemia. J Clin Microbiol, v. 45, p. 1843-1850, 2007.

90. VAZQUEZ, J.A. et al. Nosocomial acquisition of Candida albicans: an epidemiologic study. J Infect Dis, v.168, p. 195-201, 1993.
91. VISCOLI C. et al. Candidemia in cancer patients: a prospective, multicenter surveillance study by the invasive fungal infection group (IFIG) of the European Organization for research and treatment of cancer (EORTC). Clin Infect Dis 1999;28:1071-1079.

92. VOSS, A.; MEIS, J.F.; HOOGKAMP-KORSTANJE, J.A. fluconazole in the management of fungal urinary tract infections. Infection, v. 22, p. 247-251, 1994.

93. WENZEL, R.P. The economics of nosocomial infections. J Hosp Infect, v. 31, p. 79-87, 1995.

94. WHITEWAY, M.; OBERHOLZER, U. Candida morphogenesis and host-pathogen interactions. Curr Opin Microbiol, v. 7, p. 350-357, 2004.

95. WILSON, L.S. et al. The direct cost and incidence of systemic fungal infections. Value Health, v. 5, p. 26-34, 2002.

96. WINGARD, J.R. Importance of Candida species other than $\mathrm{C}$. albicans as pathogens in oncology patients. Clin Infect Dis, v. 20, p. 115-125, 1995.

97. WISPLINGHOFF, H. et al. Nosocomial bloodstream infections in US hospitals: analysis of 24,179 cases from a prospective nationwide surveillance study. Clin Infect Dis, v. 39, p. 309-337, 2004.

98. XIE, G-H. et al. Impact of invasive fungal infection on outcomes of severe sepsis: a multicenter matched cohort study in critically ill surgical patients. Critical Care, v. 12, p. R5, 2008.

99. ZAOUTIS, T.E. et al. The epidemiology and attributable outcomes of candidemia in adults and children hospitalized in the United States: a propensity analysis. Clin Infect Dis, v. 41, p. 1232-1239, 2005. 